\title{
Broadband microwave sub-second pulsations in an expanding coronal loop of the 2011 August 10 flare
}

\author{
H. Mészárosová ${ }^{1}$, J. Rybák$^{2}$, L. Kashapova ${ }^{3}$, P. Gömöry ${ }^{2}$, S. Tokhchukova ${ }^{4}$, and I. Myshyakov ${ }^{3}$ \\ 1 Astronomical Institute of the Academy of Sciences of the Czech Republic, 25165 Ondřejov, Czech Republic \\ e-mail: hana@asu.cas.cz \\ 2 Astronomical Institute of the Slovak Academy of Sciences, 05960 Tatranská Lomnica, Slovak Republic \\ 3 Institute of Solar-Terrestrial Physics SB RAS, 664033 Irkutsk, Russia \\ 4 Special Astrophysical Observatory of RAS, 196140 St. Petersburg Department, Russia \\ Received 30 December 2015 / Accepted 6 July 2016
}

\begin{abstract}
Aims. We studied the characteristic physical properties and behavior of broadband microwave sub-second pulsations observed in an expanding coronal loop during the GOES C2.4 solar flare on 2011 August 10.

Methods. The complex microwave dynamic spectrum and the expanding loop images were analyzed with the help of SDO/AIA/HMI, RHESSI, and the STEREO/SECCHI-EUVI data processing software, wavelet analysis methods, the GX Simulator tool, and the NAFE method.

Results. We found sub-second pulsations and other different burst groups in the complex radio spectrum. The broadband (bandwidth about $1 \mathrm{GHz}$ ) sub-second pulsations (temporal period range $0.07-1.49 \mathrm{~s}$, no characteristic dominant period) lasted $70 \mathrm{~s}$ in the frequency range 4-7 GHz. These pulsations were not correlated at their individual frequencies, had no measurable frequency drift, and zero polarization. In these pulsations, we found the signatures of fast sausage magnetoacoustic waves with the characteristic periods of 0.7 and $2 \mathrm{~s}$. The other radio bursts showed their characteristic frequency drifts in the range of $-262-520 \mathrm{MHz} \mathrm{s}^{-1}$. They helped us to derive average values of $20-80 \mathrm{G}$ for the coronal magnetic field strength in the place of radio emission. It was revealed that the microwave event belongs to an expanding coronal loop with twisted sub-structures observed in the 131, 94, and 193 $\AA$ SDO/AIA channels. Their slit-time diagrams were compared with the location of the radio source at $5.7 \mathrm{GHz}$ to realize that the EUV intensity of the expanding loop increased just before the radio source triggering. We reveal two EUV bidirectional flows that are linked with the start time of the loop expansion. Their positions were close to the radio source and propagated with velocities within a range of $30-117 \mathrm{~km} \mathrm{~s}^{-1}$.

Conclusions. We demonstrate that periodic regime of the electron acceleration in a model of the quasi-periodic magnetic reconnection might be able to explain physical properties and behavior of the sub-second pulsations. The depolarization process of the microwave emission might be caused by a plasma turbulence in the radio source. Finally, the observed EUV flows might be linked with reconnection outflows.
\end{abstract}

Key words. Sun: flares - Sun: corona - Sun: radio radiation - Sun: UV radiation - Sun: oscillations

\section{Introduction}

The fine structures present in the flare radio emissions are studied because these phenomena might be an effective diagnostics of processes in flare plasma. Of the various fine structures observed in radio waves (e.g., Jiřička et al. 2001), pulsations have been the subject of many papers, see the reviews, for instance, by Aschwanden $(2003,2004)$ and Nakariakov \& Melnikov (2009).

Generally, radio emissions can be classified into coherent and incoherent emission mechanisms (Aschwanden 2004). Incoherent emission results from continual processes such as thermal particle distributions that produce free-free emission (bremsstrahlung) in microwave and $\mathrm{mm}$ wavelengths for low magnetic field strengths and mildly relativistic electron distributions that generate gyrosynchrotron emission, which is naturally produced during flares. Coherent emission reflects kinetic instabilities from particle distributions. The most natural ways to produce these anisotropic particles are velocity dispersion, which creates electron beams and thus plasma emission, and mirroring in magnetic traps, which produces loss-cone instabilities (electron-cyclotron emission). The most of coherent flare-related radio emissions are driven by bursty magnetic reconnection processes and the associated flare plasma dynamics.

Pulsations with period $P>1 \mathrm{~s}$ are frequently observed (e.g., Aschwanden 2003). Nevertheless, there are also several studies of sub-second pulsations, for example, in the period range $0.025-0.055 \mathrm{~s}$ (Xie et al. 2003; Ma et al. 2003), $0.1 \mathrm{~s}$ (Karlicky et al. 2010), 0.07-0.08 s (Fleishman et al. 1994), and $0.16-0.18 \mathrm{~s}$ (Fu et al. 1990). There are rather exceptional observations at microwave frequencies (e.g., in the frequency range $5380-6250 \mathrm{MHz}$ in Fu et al. 1990).

These sub-second pulsations are typically observed at individual single frequencies, where we can recognize spikes (Fleishman \& Melnikov 1998; Fleishman 2004). On the other hand, we need to see radio dynamic spectra to recognize the type of the fine structures with the sub-second pulsating phenomena, for instance, type III pulses in Meshalkina et al. (2012).

Another problem is that radio dynamic spectra observed during solar flares can be very complex and show different types of bursts and fine structures. One possibility of studying these fine structures in detail is the separation method (Mészárosová et al. 2011a), which is based on the wavelet analysis techniques. 
This method splits an original complex (radio) spectrum into two (or more) simpler dynamical spectra according to the temporal, frequency, and spatial components of individual bursts to simplify the analysis. This method is suitable when the original radio spectrum consists of a mixture of different fine structures or bursts that are observed at the same frequencies and during the same time interval, and when it is therefore difficult to recognize individual temporal or frequency components from each other. The method also works well when weak bursts of the spectrum coincide with strong ones (then we can typically see only the strong component, while the weak one remains hidden) and when we wish to detect or locate possible fast sausage magnetoacoustic waves.

The properties of the impulsively generated sausage magnetoacoustic waves propagating along their waveguide (e.g., loops) were theoretically predicted by Roberts et al. (1983, 1984). Each of these fast sausage magnetoacoustic waves form wave trains that propagate along the waveguide. The time evolution of these trains forms a tadpole wavelet pattern with a narrow tail that precedes a broadband head (Nakariakov et al. 2004). The start of the wave decay phase corresponds to the tadpole head maximum.

In solar radio observations, these wavelet tadpoles were recognized in the gyrosynchrotron radio bursts, for example (Mészárosová et al. 2009a; tadpoles detected at the same time throughout the whole frequency range), in dm radio fiber bursts generated by the plasma emission processes (Mészárosová et al. 2009b, 2011b; slowly drifting tadpoles corresponding to the frequency drift of the whole group of fiber bursts), and in sources of narrowband dm radio spikes (Karlický et al. 2011). Mészárosová et al. (2013) found these fast magnetoacoustic waves to propagate in the fan structure of the coronal magnetic null point. These studies were supported by MHD numerical simulations conducted by Jelínek \& Karlický (2012), Pascoe et al. (2013), and Mészárosová et al. (2014), for instance.

Thus, the results of the separation method, the analysis of individual observed bursts, and MHD numerical models for the fast magnetoacoustic wave trains can help us to interpret our observational data and estimate flare plasma parameters. The goal of our study is to find a possible explanation of the broadband microwave sub-second pulsations in a dynamic spectrum that was obtained during the 2011 August 10 GOES C2.4 solar flare.

This paper is organized as follows. In Sect. 2 we analyze the observed microwave dynamic spectrum with broadband subsecond pulsations to determine their characteristic physical properties. In Sect. 3 we study flare loops belonging to this radio event with the help of imaging data (SDO/AIA, SDO/HMI, RHESSI, and STEREO/SECCHI-EUVI). Finally, a discussion and conclusions are presented in Sect. 4.

\section{Analysis of the radio dynamic spectrum with microwave sub-second pulsations}

The solar microwave event was observed at 9:33:57-9:35:07 UT on 2011 August 10 during the GOES C2.4 flare that occurred in the active region NOAA 11236 . This event was simultaneously observed with the RATAN-600 (Pariiskii et al. 1976), the Siberian Solar Radio Telescope (SSRT; Grechnew et al. 2003), and the Badary Broadband Microwave Spectropolarimeter (BBMS; Zhdanov \& Zandanov 2011, 2015).

The spectral and polarization high-resolution receiver system of the RATAN-600 was upgraded in 2010, and the current resolution is $0.014 \mathrm{~s} \mathrm{(Bogod} \mathrm{et} \mathrm{al.} \mathrm{2011).} \mathrm{This} \mathrm{telescope} \mathrm{provides} \mathrm{1D}$ spatial resolution observations that allow us to determine only
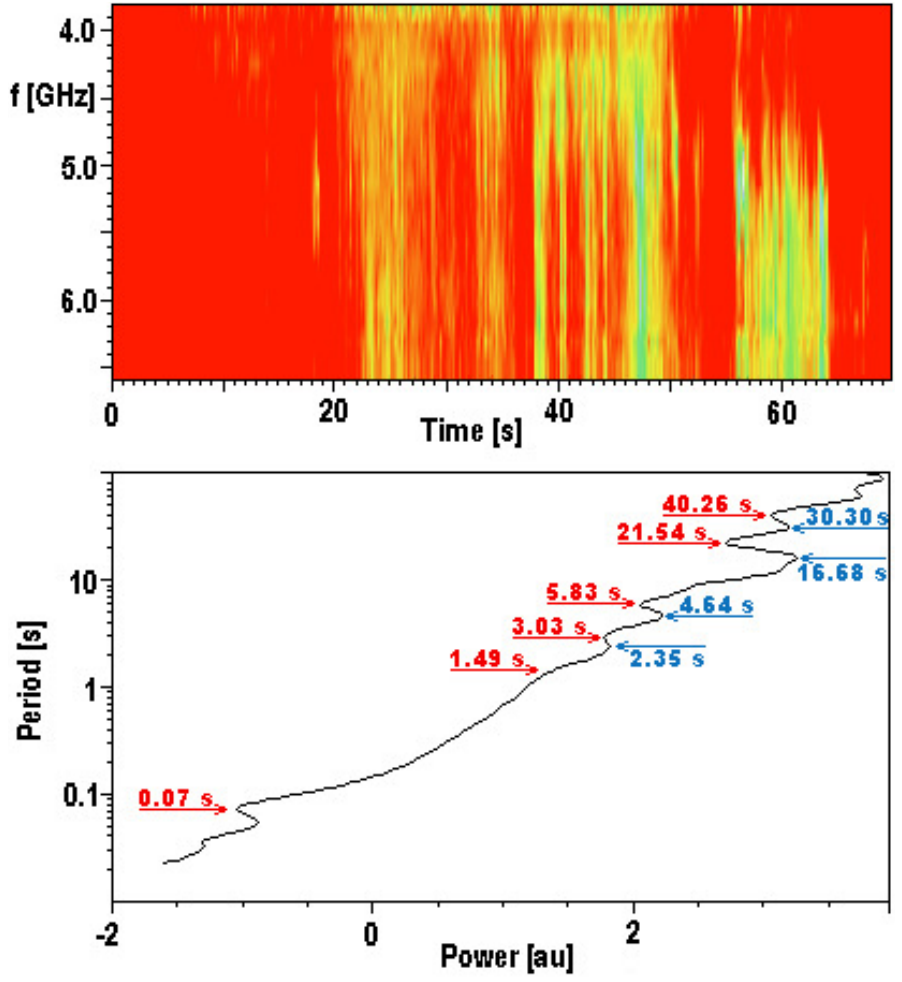

Fig. 1. Top: original complex radio dynamic spectrum with broadband pulsations that lasted 70 s (9:33:57-9:35:07 UT) and was observed by the Badary Broadband Microwave Spectropolarimeter. Bottom: averaged global wavelet spectrum (AGWS) made from the spectrum in panel a) with strong peaks at periods $P=2.35,4.64,16.68$, and $30.30 \mathrm{~s}$ (blue arrows) and with local minima at periods 0.07, 1.49, 3.03, 5.83, 21.54 , and $40.26 \mathrm{~s}$ (red arrows).

the $x$-coordinate of the flaring source. Generally, the source position can be determined with the help of two different projections based on scans measured at different times. With several projections with good angle coverage and resolution, the full map of a source can be constructed using this technique (Nindos et al. 1996).

We were unable to determine the position of our sub-second pulsation source with this technique because the emission was unstable during the time at which the projections were taken. We therefore used other properties of RATAN-600 observations. The pulses were recorded by RATAN-600 while the source was passing through the motionless Gaussian-like beam of RATAN600 for about $7 \mathrm{~s}$ with $0.014 \mathrm{~s}$ time resolution. As a result, we obtained the time profile of the sub-second pulsations modulated by the RATAN-600 beam profile that is overlaid on a more stable emitting source. By comparing the RATAN-600 profile with the profile of the sub-second pulsations from the BBMS instrument, we determined the maximum response of the RATAN-600 beam with respect to the stable emitting source. Then, the position of this maximum response was considered as the $x$-coordinate of the sub-second pulsations. To obtain the $y$-coordinate, we used the SSRT data. The result is that the position of the radio source at $5.7 \mathrm{GHz}$ was at $[X, Y]=[921,256]$ arcsec (Kashapova et al. 2013a,b).

The fine structures including the sub-second pulsations were detected in the radio dynamic spectrum (Fig. 1, top panel) in the frequency range $3797-8057 \mathrm{MHz}$ observed by the BBMS. This radio dynamic spectrum was obtained with a time resolution of $0.011 \mathrm{~s}$, and the event lasted 70 s (9:33:57-9:35:07 UT). 
The BBMS data were measured with respect to the left and right circular polarizations (LCP and RCP). We realized that the LCP flux is equal to that of the RCP, meaning that the microwave event had zero polarization.

\subsection{Separation of individual bursts detected in the BBMS radio dynamic spectrum}

We analyzed the BBMS radio dynamic spectrum with respect to individual types of observed bursts and their physical parameters. The original complex radio dynamic spectrum with broadband pulsations is shown in the top panel of Fig. 1 and was observed in the frequency range $3797-8057 \mathrm{MHz}$ with a time resolution of $0.011 \mathrm{~s}$.

To reveal individual burst types that may be included in this radio dynamic spectrum, we used the separation method (Mészárosová et al. 2011a) that is based on the wavelet analysis technique. We used the temporal periods (for more details see the method description in Mészárosová et al. 2011a) to separate the original radio dynamic spectrum. We computed an average global wavelet spectrum (AWGS) from all time series of the original radio dynamic spectrum (top panel, Fig. 1). This AWGS curve is shown in the bottom panel of Fig. 1. This shows individual peaks of the AWGS with characteristic periods $P=2.35$, $4.64,16.68$, and $30.30 \mathrm{~s}$ (blue arrows) as well as individual local minima with periods of $0.07,1.49,3.03,5.83,21.54$, and $40.26 \mathrm{~s}$ (red arrows). Then we computed the new separated radio dynamical spectra, each of them for only a selected period range of the characteristic peak period. For example, for bursts with a dominant temporal period of $16.68 \mathrm{~s}$, the new radio dynamic spectrum was computed in the period range 5.83-21.54 s, which only shows the bursts of the selected dominant period (see bottom panel of Fig. 1). The resulting separated spectrum is displayed in panel $\mathrm{g}$ of Fig. 2.

An overview of all new separated dynamic spectra is presented in Fig. 2 (left panels) with separation ranges given in the figure caption. Arrows in panels $\mathrm{i}-\mathrm{k}$ show the frequency drifts of these bursts (see Table 1). The positive and negative parts of amplitudes (in relation to their mean values) are given in white and black, respectively (left panels). The period range $<0.07 \mathrm{~s}$ contains only instrument interferences (not presented here). All new separated spectra were computed with the inverse wavelet method (Torrence \& Compo 1998).

There is no dominant period (no blue arrow in Fig. 1) for the temporal scale $0.07-1.49 \mathrm{~s}$ (panel a, Fig. 2). This is the temporal scale range of the sub-second pulsations. This means that these pulsations consist of a mixture of temporal scales where no individual scale is dominant. Most pulsations displayed in panel a of Fig. 2 are broadband (about $1 \mathrm{GHz}$ ).

We computed cross-correlations for individual time series of the dynamic spectrum displayed in panel a of Fig. 2. These series are cross-correlated by $50-65 \%$, but only at the nearest frequencies to each other. This shows that the sub-second pulsations are not correlated in general.

The bursts we present in the individual left panels (Fig. 2) are not directly visible in the original radio spectrum (Fig. 1, top panel). Therefore, the validity of these separations is shown in right panels (Fig. 2). Selected individual time series of the original radio dynamic spectrum (in red) are compared with the separated one (in green) always at the same frequency of $5194 \mathrm{MHz}$. For example, separated individual peaks (in green, panel b) belong to the the finest peaks of the original spectrum, that is, to the sub-second pulsations. The selected time interval in panel b) is $49-59 \mathrm{~s}$ of the entire $70 \mathrm{~s}$ event duration to show the finest peaks of the sub-second pulsations. The separated individual peaks in panels c, e, and g reflect the different types of pulses without any measurable frequency drift. Finally, the separated peaks in panels $\mathrm{i}-\mathrm{k}$ show various types of bursts with their significant frequency drifts (arrows 1-4). Frequency drifts (arrows 1, 2, and 3 in panel i) are equal to 520,138 , and $-262 \mathrm{MHz} \mathrm{s}^{-1}$, respectively. The frequency drift (arrow 4 in panel k) is at $158 \mathrm{MHz} \mathrm{s}^{-1}$.

We used the density model of Aschwanden \& Benz (1995) with numerical values for the height of $h 1=1.6 \times 10^{2} \mathrm{Mm}$, for the density of the quiet corona $n_{Q}=4.6 \times 10^{8} \mathrm{~cm}^{-3}$, and the parameter $p=2.38$. We used this model to compute characteristic parameters of the individual radio bursts with frequency drifts in panels $\mathrm{i}$ and $\mathrm{k}$ of Fig. 2. Thus, we obtained the average electron densities at mean coronal altitudes as well as the plasma velocities.

Then we computed a magnetic field strength $B[\mathrm{G}]=v_{p}(S \rho)^{1 / 2} / 2.03 \times 10^{11}$ for individual drifting bursts, where $v_{p}$ is plasma velocity [ $\left.\mathrm{cm} \mathrm{s}^{-1}\right]$ and $S \rho$ is the starting averaged electron density $\left[\mathrm{cm}^{-3}\right]$. These characteristic parameters are shown in Table 1.

These bursts (panels i and k, Fig. 2) have their positive frequency drifts in a range of $138-520 \mathrm{MHz} \mathrm{s}^{-1}$ and only one of them shows a negative frequency drift $\left(-262 \mathrm{MHz} \mathrm{s}^{-1}\right)$. While the starting averaged electron density is of about $2 \times 10^{11} \mathrm{~cm}^{-3}$ (with one exception for the burst with negative frequency drift), the ending averaged electron density is always of about $4 \times$ $10^{11} \mathrm{~cm}^{-3}$. Similarly, the starting mean coronal altitude is of about $4 \mathrm{Mm}$ (with one exception for the burst with negative frequency drift), the ending mean coronal altitudes are always of about $3 \mathrm{Mm}$. The plasma velocity is about $90 \mathrm{~km} \mathrm{~s}^{-1}$ and the magnetic field strength is equal to $20-40 \mathrm{G}$, except for the burst (arrow 1, Fig. 2) with the plasma velocity, which equals $329 \mathrm{~km} \mathrm{~s}^{-1}$ and has a magnetic field strength of $80 \mathrm{G}$.

\subsection{Fast sausage magnetoacoustic waves detected in the separated radio dynamic spectra}

We also searched for possible fast sausage magnetoacoustic waves propagating in situ of the radio source. It is easier to find them in the time series of the separated dynamic spectra (Fig. 2) because it is much simpler to find them there than in the original dynamic spectra (Fig. 1, top panel) using the wavelet separation method (Mészárosová et al. 2011a).

We found these magnetoacoustic waves in the separated radio dynamic spectra shown in panels a and $c$ (Fig. 2). In Figs. 3 and 5 we present examples of time series and their wavelet tadpole patterns as signatures of propagating sausage magnetoacoustic waves.

We show examples of the wavelet tadpoles with the characteristic period $P \approx 0.7 \mathrm{~s}$ and the time series in the panels a-d of Fig. 3. We selected characteristic time intervals (9:34:56-9:35:01 UT) of the entire time series of the separated radio dynamic spectrum (panel a, Fig. 2) where the magnetoacoustic waves were detected. The selected time series were observed at frequencies 4887 (panel a), 5579 (panel b), 5957 (panel c), and $6073 \mathrm{MHz}$ (panel d). The series have the peak maxima (red arrows) at the time of the wavelet tadpole head maxima. The frequency drift of these head maxima is equal to $2892 \mathrm{MHz} \mathrm{s}^{-1}$.

We compared this result with the original time series since the sub-second pulsations have no measurable frequency drift despite of their high bandwidth (about $1 \mathrm{GHz}$ ). We found one exceptional case: Fig. 4 shows a detail for the time interval 

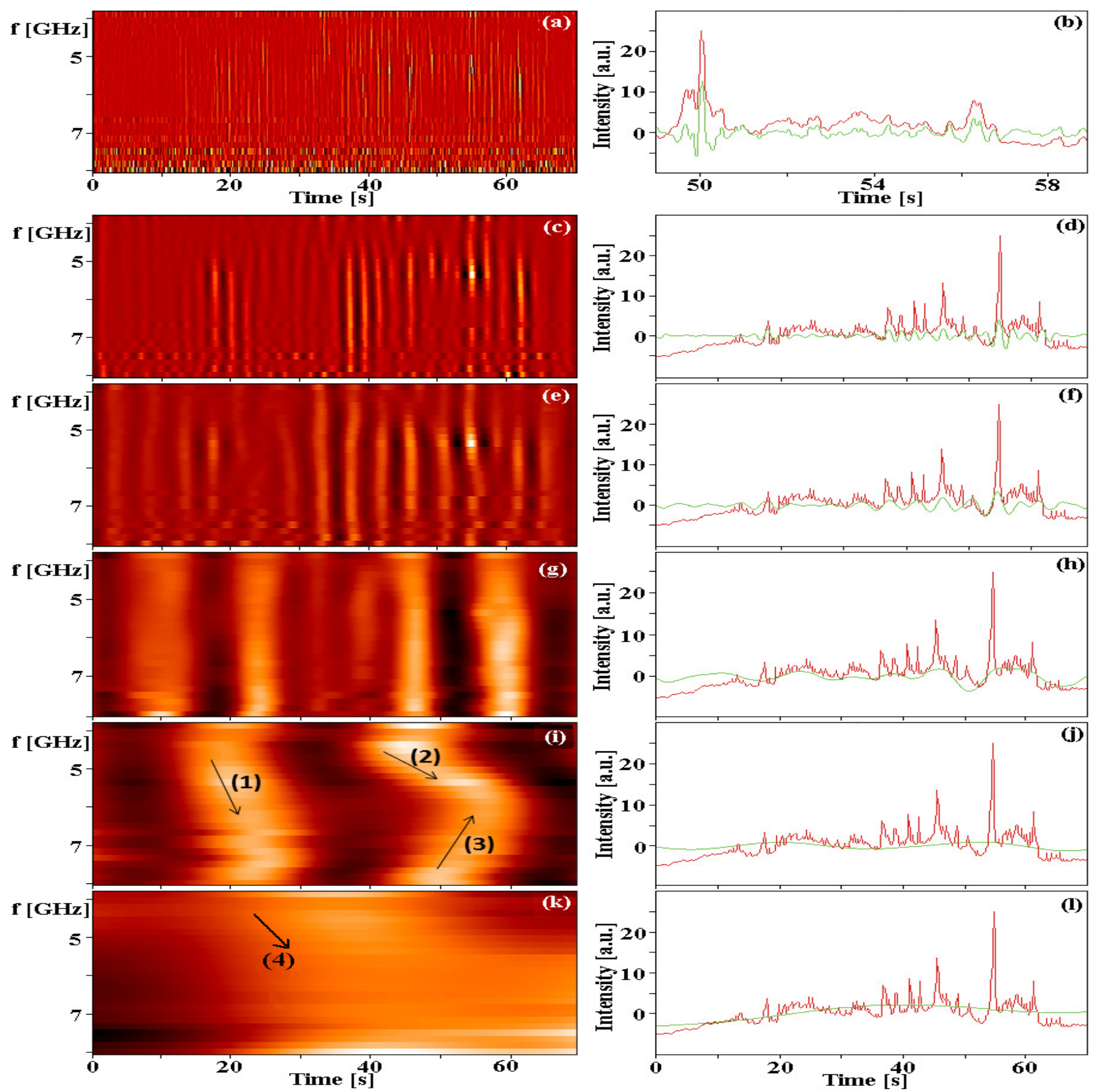

Fig. 2. Dynamic spectra of separated bursts (left panels) and their selected time series (right panels). Bursts are separated according to their characteristic period ranges of 0.07-1.49 s (panel a)), 1.49-3.03 s (panel c)), 3.03-5.83 s (panel e)), 5.83-21.54 s (panel g)), 21.54-40.26 s (panel i)), and for periods $>40.26 \mathrm{~s}$ (panel $\mathbf{k}$ )). The positive and negative parts of the amplitudes (in relation to their mean values) are given in white and black, respectively (left panels). Frequency drifts of 520,138 , and $-262 \mathrm{MHz} \mathrm{s}^{-1}$ as well as $158 \mathrm{MHz} \mathrm{s}^{-1}$ are marked by arrows in panel i (1, 2, and 3) and $\mathrm{k}$ (arrow 4). For a detailed comparison the individual peaks in the radio time series of the original radio dynamic spectrum (in red) and of the separated spectrum (in green) are shown for the selected $5194 \mathrm{MHz}$ in the right panels. To show the fine individual peaks, we selected the time interval of $49-59 \mathrm{~s}$ (in panel b)) within the entire $70 \mathrm{~s}$ event duration.

of 9:34:56-9:35:02 UT of the original radio dynamic spectrum with two pulses and with a frequency drift of $2892 \mathrm{MHz} \mathrm{s}^{-1}$, that is, with the same frequency drift as the wavelet tadpole head maxima in Fig. 3. These pulses occurred at the same frequencies as the detected magnetoacoustic waves in Fig. 3. The characteristic parameters (Table 1) of these pulses (arrow 1 in Fig. 4) are significantly different from the other drifting bursts of Fig. 2 in the frequency drift, event duration, plasma velocity, and magnetic field strength. On the other hand, such parameters as the starting and ending averaged electron density and the starting and ending mean coronal altitudes are similar for all observed events (Table 1).

Another case with detected magnetoacoustic waves is presented in Fig. 5. We show selected time series of the separated radio dynamic spectrum (panel c, Fig. 2) and their wavelet tadpole patterns with a characteristic period $P \approx 2 \mathrm{~s}$. These time series 
Table 1. Characteristic parameters of radio bursts.

\begin{tabular}{|c|c|c|c|c|c|c|c|c|c|c|c|c|c|}
\hline $\mathrm{F}$ & A & $\begin{array}{c}\mathrm{SF} \\
(\mathrm{MHz})\end{array}$ & $\begin{array}{c}\mathrm{EF} \\
(\mathrm{MHz})\end{array}$ & $\begin{array}{l}\text { ST } \\
\text { (s) }\end{array}$ & $\begin{array}{l}\text { ET } \\
(s)\end{array}$ & $\begin{array}{c}\text { Dur } \\
(\mathrm{s})\end{array}$ & $\begin{array}{c}\mathrm{FD} \\
\left(\mathrm{MHz} \mathrm{s}^{-1}\right)\end{array}$ & $\begin{array}{c}\mathrm{S} \rho \\
\left(\mathrm{cm}^{-3}\right)\end{array}$ & $\begin{array}{c}\mathrm{E} \rho \\
\left(\mathrm{cm}^{-3}\right)\end{array}$ & $\begin{array}{c}\mathrm{SA} \\
(\mathrm{Mm})\end{array}$ & $\begin{array}{c}\text { EA } \\
(\mathrm{Mm})\end{array}$ & $\begin{array}{c}v_{\mathrm{p}} \\
\left(\mathrm{km} \mathrm{s}^{-1}\right)\end{array}$ & $\begin{array}{c}B \\
(\mathrm{G})\end{array}$ \\
\hline 2 & 1 & 4 & & & & & 52 & 41,101 & (1) 101 & 4. & & 32 & 80 \\
\hline 2 & 2 & 4408 & 44 & 4 & & & 138 & 1 & $4.09 \times$ & 4. & & 8 & 21 \\
\hline 2 & 3 & 7607 & 6073 & 7 & 50 & 5.86 & -262 & 11 & $4.57 \times$ & 2. & . & 95 & 40 \\
\hline 2 & 4 & 4519 & 5872 & 24.61 & 33.16 & & 158 & & $4.28 \times 10^{11}$ & 4. & 3. & 96 & 24 \\
\hline 4 & 1 & 4887 & 5957 & 61.76 & 62.13 & 0.37 & 2892 & $2.96 \times 10^{11}$ & $4.00 \times 10^{11}$ & 3.89 & 3.29 & 1610 & 432 \\
\hline
\end{tabular}

Notes. $\mathrm{F}=$ figure number; $\mathrm{A}=$ arrow number; $\mathrm{SF} \& \mathrm{EF}=$ starting and ending frequency; $\mathrm{ST} \& \mathrm{ET}=$ starting and ending time; Dur $=$ duration; $\mathrm{FD}=$ frequency drift; $\mathrm{S} \rho \& \mathrm{E} \rho=$ starting and ending averaged electron density; $\mathrm{SA} \& \mathrm{EA}=$ starting and ending mean coronal altitudes; $v_{\mathrm{p}}=$ plasma velocity; and $B=$ magnetic field strength.
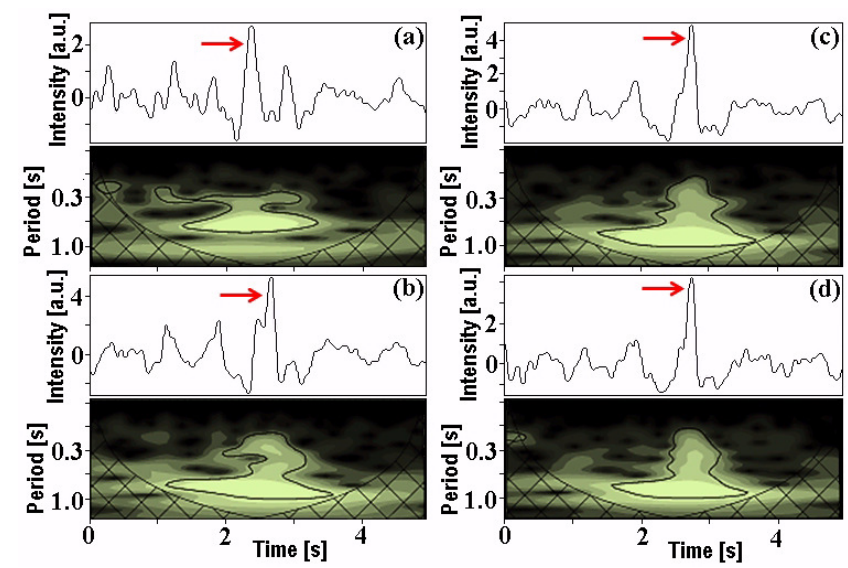

Fig. 3. Selected time series of the separated radio dynamic spectrum from panel 1a (Fig. 2) and the wavelet tadpole patterns with a characteristic period $P \approx 0.7$ s. These time series (9:34:56-9:35:01 UT) observed at frequencies 4887 (panel a)), 5579 (panel b)), 5957 (panel c)), and $6073 \mathrm{MHz}$ (panel d)) show their peak maxima (red arrows) equal to the maxima of the wavelet tadpole heads. The frequency drift of these head maxima is $2892 \mathrm{MHz} \mathrm{s}-1$.

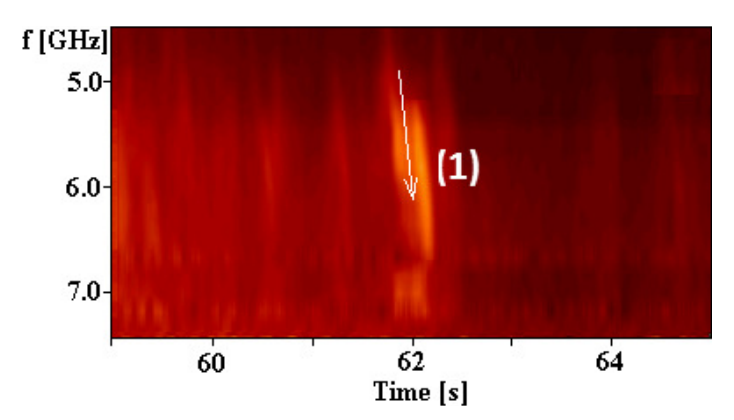

Fig. 4. Detail (9:34:56-9:35:02 UT) of the original radio dynamic spectrum (panel a) of Fig. 1) with two close pulses with an average frequency drift of $2892 \mathrm{MHz} \mathrm{s}^{-1}$.

(9:34:51-9:35:07 UT) observed at frequencies 5026 (panel a), 5398 (panel b), 5579 (panel c), and $6593 \mathrm{MHz}$ (panel d) have their peak maxima (red arrows) equal to the maxima of the wavelet tadpole heads. There is no measurable frequency drift of these tadpole head maxima in agreement with no f-drift of the bursts in panel c (Fig. 2).
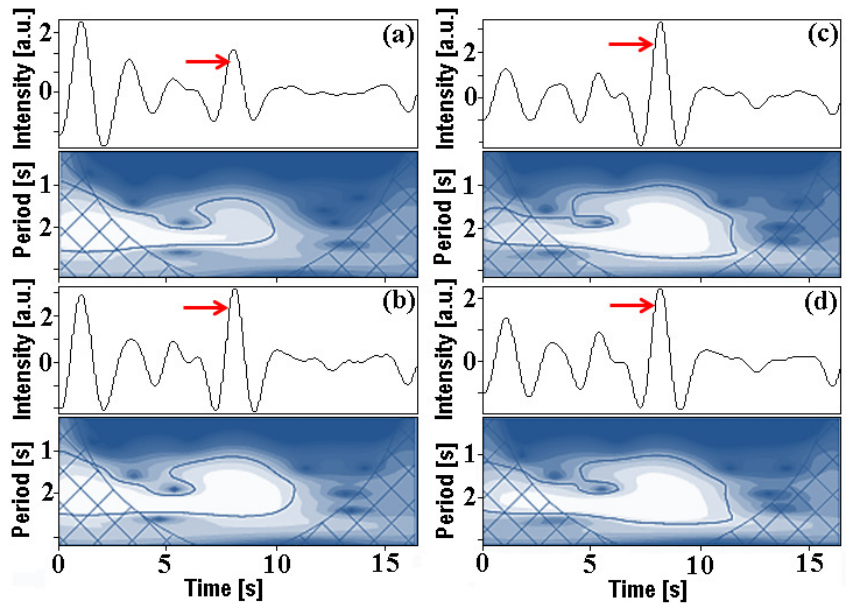

Fig. 5. Selected time series of separated radio dynamic spectrum in panel 2a (Fig. 2) and the wavelet tadpole patterns with a characteristic period $P \approx 2$ s. These time series (9:34:51-9:35:07 UT) observed at frequencies 5026 (panel a)), 5398 (panel b)), 5579 (panel c)), and $6593 \mathrm{MHz}$ (panel d)) show their peak maxima (red arrows) equal to the maxima of the wavelet tadpole heads. There is no measurable frequency drift of these tadpole head maxima.

\section{Analysis of the expanding flare loop in the imaging data}

To analyze flaring loops and their near environment related to the event we studied, we used imaging data with high spatial resolution acquired by these instruments: (i) EUV images observed by the Atmospheric Imaging Assembly onboard the Solar Dynamics Observatory (SDO/AIA; Lemen at al. 2012); (ii) magnetograms observed by the Helioseismic and Magnetic Imager (SDO/HMI; Schou et al. 2012; Scherrer et al. 2012); (iii) X-ray data of the Reuven Ramaty High-Energy Solar Spectroscopic Imager (RHESSI; Lin et al. 2002), and (iv) EUV images observed by the Extreme Ultraviolet Imager onboard the Solar TErrestrial RElations Observatory (STEREO/SECCHI-EUVI; Wuelser et al. 2004). These imaging data were reduced according to the available instrument manuals using the standard software tools provided within the SolarSoft package (Freeland \& Handy 1998).

\subsection{Analysis of the SDO/AIA and SDO/HMI imaging data}

We searched for our event in all SDO/AIA channels and found that the event loops and their footpoints were visible only in the 131,94 , and $193 \AA$ channels, while they cannot be 


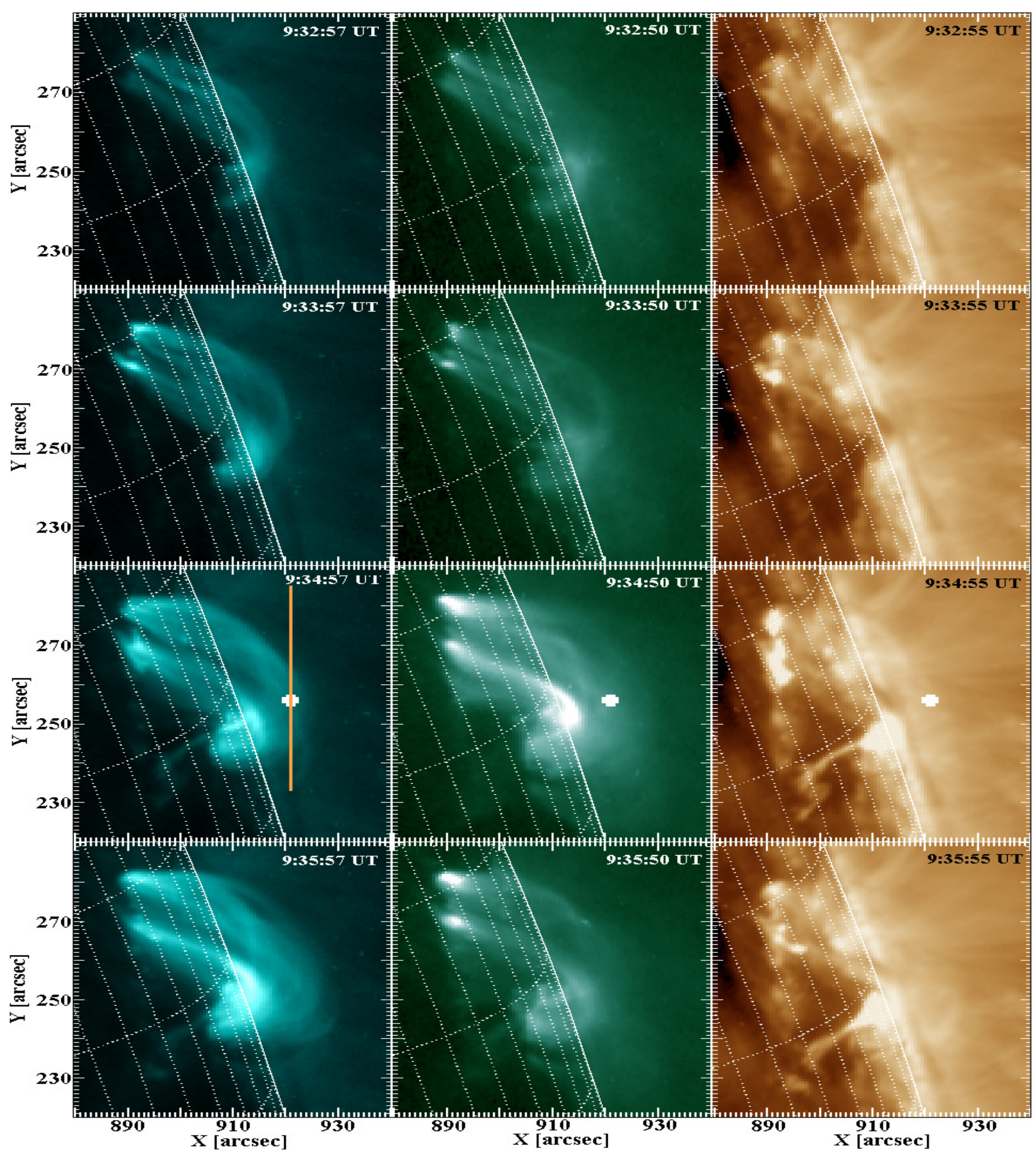

Fig. 6. Time evolution (9:32-9:35 UT) of expanding loops observed by SDO/AIA in channels $131 \AA$ (left column), $94 \AA$ (middle column), and $193 \AA$ (right column). The location of the radio source at $5.7 \mathrm{GHz}$ is indicated by a white blob at 9:34 UT (third row). An example of the slit position passing the radio source is shown by an orange vertical solid line in the left column (131 $\AA$ ) for the 9:34:57 UT panel.

found in the other channels, including the 211 and $335 \AA$ channels. Following the primary ions and the corresponding temperatures of the individual AIA channels (Table 1 in Lemen et al. 2012), we can conclude that the emission comes from the hot flare plasma in the temperature range of characteristic $\log (T)=6.8(94 \AA), 7.0$ (131 , Fe XXI sensitivity peaks) and 7.3 (193 A, Fe XXIV ion sensitivity peak) and that there was no emission for the temperatures below $\log (T)=6.5(335 \AA$, $211 \AA$ ). In the $193 \AA$ channel the loops are only very weakly seen, except for the loop footpoints.

The time evolution (9:32-9:35 UT) of the expanding loops observed by SDO/AIA in channels $131 \AA$ (left column), $94 \AA$ (middle column), and $193 \AA$ (right column) is shown in Fig. 6. This SDO/AIA event consists of (seemingly) one group 
of loops at the beginning of the evolution ( 9:32 UT). Later (after $\sim 9: 33$ UT) another loop is visible that expands toward higher altitudes. This set of two loops (expanded and nonexpanded) shows the highest EUV emissivity of the hot flare plasma $(\log (T)=7.0)$ at about 9:34 UT and later (see Fig. 7).

The location of the radio source at $5.7 \mathrm{GHz}$ is indicated by a white blob at 9:34 UT (third row in Fig. 6). This shows that the radio source (studied in Sect. 2) was observed at the time of the increased EUV emission visible in the 131 and $94 \AA$ and when it was localized in the expanding loop.

We studied the 131,94 , and $193 \AA$ data in more detail with the help of time series (temporal evolution during interval 9:31:33-9:39.57 UT) derived for the individual vertical slits of the fix position in the axis $Y(233-286$ arcsec) and of different $X$-axis positions in the range of $881-928$ arcsec with a step of 1 arcsec. An example of the slit position passing the radio source is presented by the orange vertical solid line in the left column (131 A) for 9:34:57 UT panel (Fig. 6).

An example of the time-slit diagram for the SDO/AIA $131 \AA$ channel for the time interval 9:31:33-9:39.57 UT and the slit at the position $X=921$ arcsec and $Y=233-286$ arcsec is presented in Fig. 7. Panel a) shows the original time-slice diagram with the EUV emission that started at a time of about 9:34 UT at a coordinate about $Y=255$ arcsec. The highest intensity is displayed after 9:37 UT at $Y \approx 250-265$ arcsec.

The position and temporal extent (9:33:57-9:35:07 UT) of the radio source, observed at $5.7 \mathrm{GHz}$, is shown with the horizontal solid line in the individual panels of Fig. 7.

The individual time-slit temporal structures in the AIA $131 \AA$ channel emission were separated according to their characteristic temporal scales using the wavelet separation method of Mészárosová et al. (2011a). The separated emissions are shown in Fig. 7. Panels b, c, and d show separated temporal structures revealed at their characteristic temporal scales of 42-120 s, 120-348 s, and scales >348 s, respectively. Different $131 \AA$ flows are shown in panels b) and c). These bidirectional flows start before the radio emission begins $(\approx 9: 33$ UT) and are located at the $Y$-coordinate of the radio source $(Y=256 \operatorname{arcsec})$. Then the flows propagate toward the coordinates $Y=280$ and $Y=240$ arcsec. The individual arrows $1,2,3$, and 4 (Fig. 7) show different plane-of-sky flow velocities along the loop with values of $117,109,30$, and $70 \mathrm{~km} \mathrm{~s}^{-1}$, respectively. The flow displayed in panel b seems to be connected mainly with the radio source (in time as well as in place). The flows in panel c might be connected with the radio source as well as the loops footpoints. Panel d shows the $131 \AA$ emission, which starts about the time and in the approximate location of the radio source and propagates in a similar way as the flows in panel c. The highest emission intensity is detected after 9:38 UT and later (beyond the scope of our study).

The time-slice diagrams at different slit positions around $X=921$ arcsec are similar to the time-slice diagram presented in Fig. 7. Similar results were also obtained for the $94 \AA$ channel. We detected no kink oscillations in our time-slice diagrams.

We also used SDO/HMI data because of the situation of the magnetic field lines of flaring loops with respect to their characteristic values of magnetic field strength. These SDO/HMI data were processed using the GX Simulator tool (Nita et al. 2015) to reconstruct a three-dimensional two-loop model of the magnetic field line configuration. This model was obtained from a potential extrapolation based on the SDO/HMI data of the event. The panels in Fig. 8 show reconstructed approximate magnetic field lines of 20-40 G (panel a), 80 G (panel b), and $450 \mathrm{G}$ (panel c).

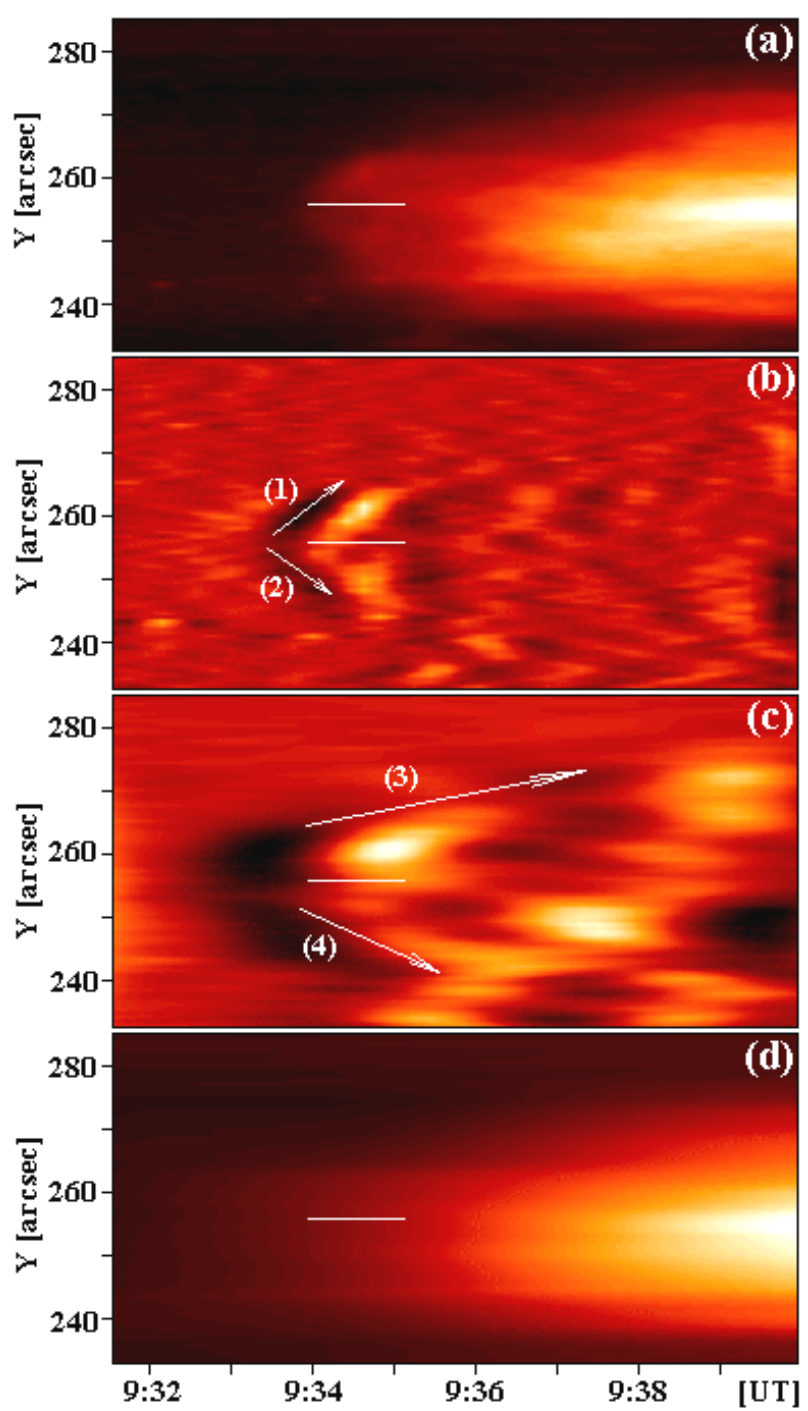

Fig. 7. Example of time-slit diagram (9:31:33-9:39:57 UT) for the SDO/AIA $131 \AA$ channel and slit at position $X=921 \mathrm{arcsec} / Y=233-286 \operatorname{arcsec}$. Panel a): original time-slice diagram. Separated time structures according to their characteristic temporal scales $42-120 \mathrm{~s}, 120-348 \mathrm{~s}$, and $>348 \mathrm{~s}$ are shown in panels $\mathbf{b}), \mathbf{c})$, and $\mathbf{d})$, respectively. The position $(Y=256 \mathrm{arcsec})$ and radio source time interval $(9: 33: 57-9: 35: 07$ UT) is presented by the horizontal solid line in individual panels. Individual arrows mark detected EUV flows propagating in the loop area. Estimated velocities are $117,109,30$, and $70 \mathrm{~km} \mathrm{~s}^{-1}$ for arrows $1,2,3$, and 4, respectively.

While these magnetic field lines of a weak magnetic field are rather parallel, dense, and concentrated mainly around the expanded loop (panel a), the magnetic field lines of the stronger magnetic field are rather less structured and more dispersed throughout the entire active area (panels $b-c)$.

We used the magnetic field extrapolation in the potential approach in our study because the limb effects are strong in the reconstructed region. This is not a fully correct description of the magnetic field characteristics during the flare. Several indicators make this suitable, however: (1) the magnetic field lines shown in Fig. 8 and the flare loops seen in EUV (Fig. 6) agree well. (2) The reconstructed magnetic field lines obtained by the nonlinear force-free approach using an optimization method (Rudenko \& Myshyakov 2009) also agree well, but in this case we cannot see fine details (twisted magnetic field lines) because of the limb effects. (3) The results agree well with the theoretical 


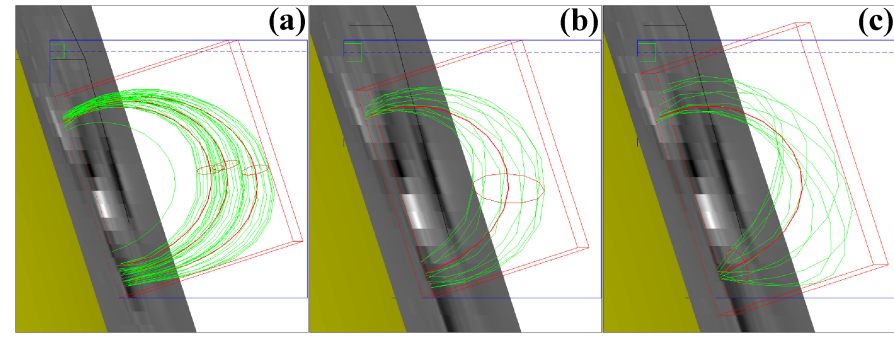

Fig. 8. Three-dimensional two-loop model of the magnetic field line configuration obtained from a potential extrapolation in the GX Simulator. The panels show reconstructed magnetic field lines of $20-40 \mathrm{G}$ (panel a)), 80 G (panel b)), and 450 G (panel c)).

modelling for a gyrosynchrotron model of the radio emission, for instance (Kashapova et al. 2013a).

\subsection{RHESSI and STEREO/SECCHI-EUVI imaging data of the loops}

We compared RHESSI and STEREO/SECCHI-EUVI observations with the SDO/AIA data to obtain more information about the active area.

However, as a first step, the individual loops observed by SDO/AIA in channels 131,94 , and $193 \AA$ at 9:34 UT were processed by the noise adaptive fuzzy equalization method (NAFE; Druckmüller 2013), and the result is shown in Fig. 9. The NAFE method allows us to see the individual loop sub-structures better (compare the panels of Fig. 9 with those in the third row in Fig. 6). Thus, the panel for $131 \AA$ (Fig. 9) shows that the expanding loop might consist of about three twisted individual sub-structures that possibly cross each other. The non-expanding loop possibly consists of two parallel loops in reality. Positions of the main loop footpoints are marked by crosses A-D in the middle panel. It seems that the expanding and non-expanding loops are placed between the footpoints $\mathrm{A}$ and $\mathrm{C}$ and $\mathrm{B}$ and $\mathrm{D}$, respectively.

Each panel in Fig. 9 is overlaid by RHESSI X-ray sources in the 3-6 keV band, $6-12 \mathrm{keV}$ band, and $25-50 \mathrm{keV}$ band. All panels of Fig. 9 show that the highest intensity of all X-ray bands is focused at the loop footpoints and below the expanding loop.

We compared the SDO/AIA and STEREO/SECCHI-EUVI observations. The time evolution of the individual observed flare structures (flare ribbons) is shown in Fig. 10. The SDO/AIA (panels a-d) and STEREO/SECCHI-EUVI (panels e-h) observations are in the channels $193 \AA$ (panels a-b), $304 \AA$ (panels c-d), $195 \AA$ (panels e-f), and $304 \AA$ (panels g-h). The panels in the left column reflect the situation before the flare (9:26-9:31 UT), and the panels in the right column the situation after the flare (9:35-9:36 UT). The formation of a new flare structure visible between the older ones (arrows 1 and 2) is marked by arrow 3 .

To identify these particular footpoint positions detected in the SDO/AIA images (Fig. 9) in the STEREO/SECCHI-EUVI images (Fig. 10) we used transformations based on the coordinate system definitions for solar images (Thompson 2006), the dedicated routines (see, e.g., Thompson \& Wei 2010) from the SolarSoft package (Freeland \& Handy 1998), and information stored in the FITS data file headers. In particular, images taken at 09:39:50 UT and 09:35:30 UT by the SDO/AIA and STEREO/SECCHI-EUVI instruments were used. We computed the positions of footpoints $\mathrm{A}-\mathrm{D}$ for different values of radial heights in a range $0-5000 \mathrm{~km}$ with a step of $100 \mathrm{~km}$. In all these

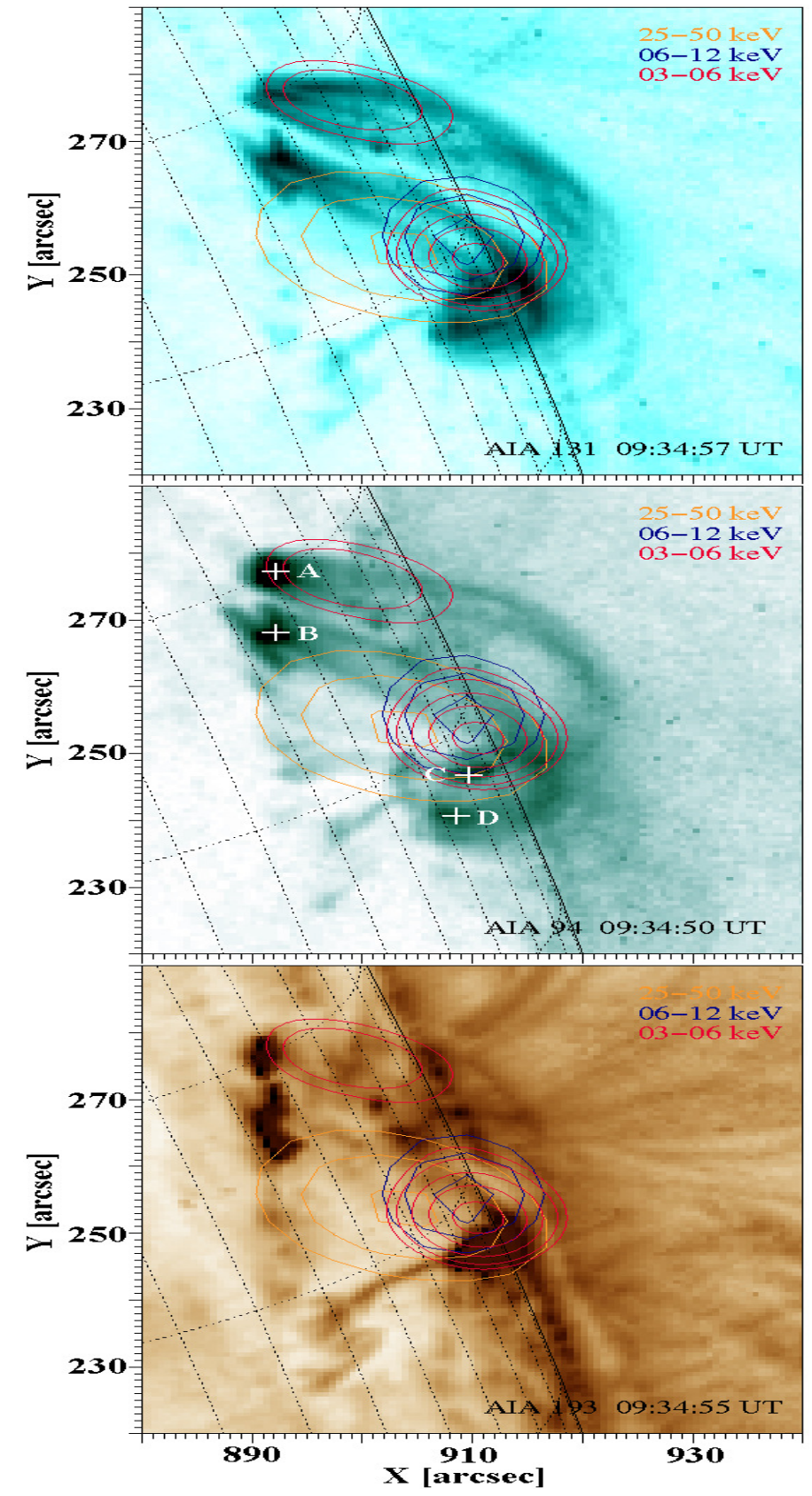

Fig. 9. Individual loops observed by SDO/AIA in channels 131, 94, and $193 \AA$ at 9:34 UT processed with the NAFE method. Each panel is overlaid by RHESSI X-ray sources in different energy bands shown as contours: $03-06 \mathrm{keV}$ (in red), $06-12 \mathrm{keV}$ (in blue), and $25-50 \mathrm{keV}$ (in yellow). The integration time was 9:34:00-9:35:00 UT for the 3-6 keV band and 9:34:20-9:35:00 UT for the 6-12 and 25-50 keV bands. Individual contours are displayed for 65,82 , and $98 \%(25-50 \mathrm{keV}$ band), 79, 87, and 95\% (6-12 keV band), and for $47,56,69,82$, and $95 \%$ (3-6 keV band) of maxima in their intensity. Loop footpoints are marked by crosses A-D in middle panel.

values we searched for those that might be connected with the postflare ribbons.

The resulting footpoint positions in the STEREO/SECCHIEUVI images are shown in panel $\mathrm{f}$ of Fig. 10. The positions of footpoints A, B, C, and D are indicated by crosses at the radial heights of $1.4,1.5,3.5$, and $2.3 \mathrm{Mm}$, respectively. The footpoints computed for other values of the radial heights were outside of the ribbons under study. Footpoints A and B are connected 

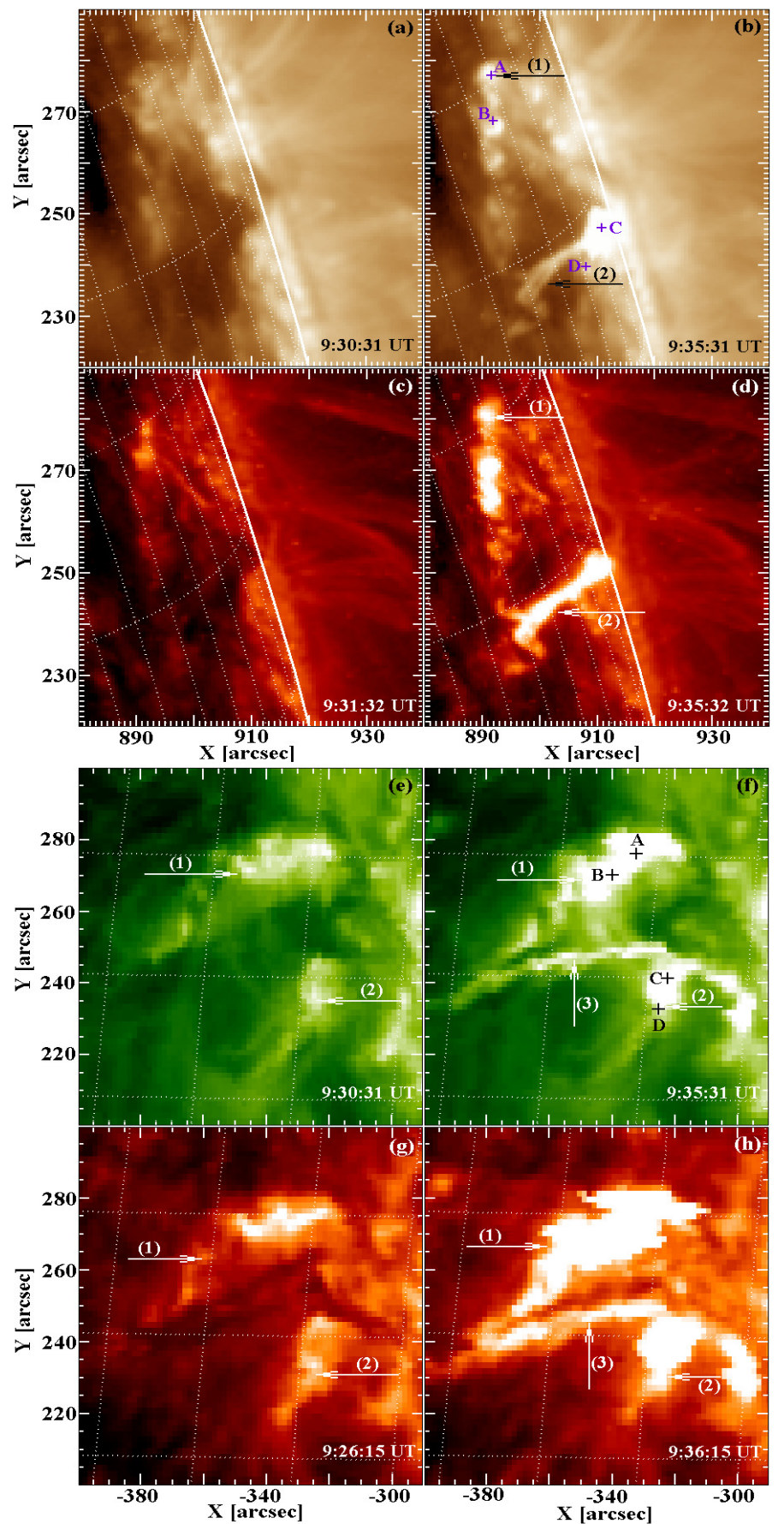

Fig. 10. Time evolution of individual flare structures (footpoint ribbons) observed by SDO/AIA (panels a)-d)) and STEREO/SECCHI-EUVI (panels e)-h)). Observations are provided at channels $193 \AA$ (panels a)-b)), $304 \AA$ (panels c)-d)), $195 \AA$ (panels e)-f)), and $304 \AA$ (panels $\mathbf{g})-\mathbf{h})$ ). Panels in the left and right columns reflect the situation before (9:26-9:31 UT) and after (9:35-9:36 UT) formation of the new flare structure (arrow 3 ) that is visible between the older ones marked by arrows 1 and 2. Positions of individual loop footpoints (see middle panel in Fig. 9) are marked by crosses A-D in panels b) and f).

with the part of the ribbon (coordinate $Y \approx 270-280$ arcsec) where higher EUV intensity was observed. Moreover, these footpoints were detected with a similar radial height (1.4 and $1.5 \mathrm{Mm}$ ). Footpoint $\mathrm{C}$ is connected with the part of the ribbon (coordinate $Y \approx 240$ arcsec) with the highest EUV intensity observed by both the SDO/AIA and the STEREO/SECCHI-EUVI

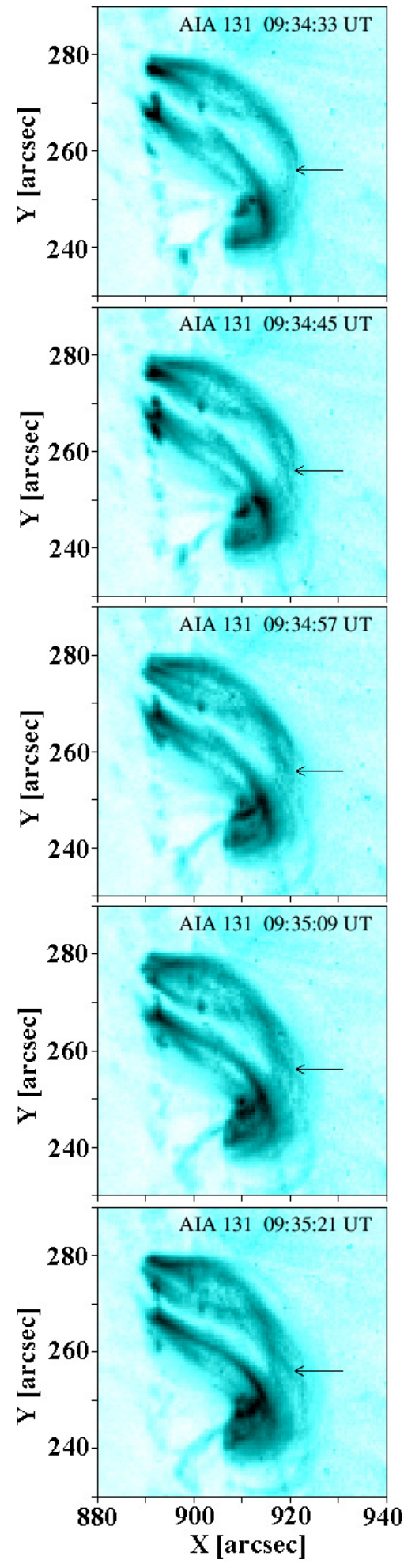

Fig. 11. Fine sub-structures of the expanding loop observed by SDO/AIA in 131 Å channel during 9:34:33-9:35:21 UT processed with the NAFE method. The position of the radio source is marked by the arrow.

instruments. Footpoint $\mathrm{D}$ is localized below this part of the ribbon (coordinate $Y \approx 230 \operatorname{arcsec}$ ).

\section{Discussion and conclusions}

We studied the solar microwave event observed at 9:33:57-9:35:07 UT by the Badary Broadband Microwave 
Spectropolarimeter on 2011 August 10 during the GOES C2.4 flare, which occurred in the active region NOAA 11236. The position of the background flaring radio source at $5.7 \mathrm{GHz}$ was obtained using the SSRT and RATAN-600 observation at this radio frequency (Kashapova et al. 2013a,b).

We revealed that the microwave radio dynamic spectrum (Fig. 1, top panel) observed in the frequency range $(3797-8057 \mathrm{MHz})$ with zero polarization consists of broadband (about $1 \mathrm{GHz}$ ) sub-second pulsations and other different bursts (Fig. 2). Some bursts have frequency drifts in the range $-262-2892 \mathrm{MHz} \mathrm{s}^{-1}$ (Table 1). We derived values of the magnetic field strength in the range $21-432 \mathrm{G}$ for these bursts with the frequency drift.

The signatures (wavelet tadpole patterns) of fast sausage magnetoacoustic waves propagating in situ of the radio source were detected in our data. In the first case (Fig. 3), the wave characteristic period is $0.7 \mathrm{~s}$ and the frequency drift of the tadpole head maxima is $2892 \mathrm{MHz} \mathrm{s}^{-1}$, which is the same as the frequency drift of the pulses (Fig. 4) observed in the same time as the magnetoacoustic waves. In the second case (Fig. 5), the wave characteristic period is about $2 \mathrm{~s}$ and there is no frequency drift of the tadpole head maxima.

According to the SDO/AIA 131 and $94 \AA$ images, only two seemingly simple and faint loops were observed near to the limb before 9:32:21 UT. From this moment, one of the loops (on the higher $Y$-coordinate values) expanded toward higher altitudes. The second loop remained more or less at its original position. The expanding loop spread to an increasingly greater area, and new loop sub-structures were created here eventually. This raises the question why just this one loop of the two expanded. A possible reason for this situation can be seen in the STEREO/SECCHI-EUVI top point of view of the active region shown in Fig. 10. This shows the situation (left column) of two very weak footpoint ribbons that are indicated with arrows 1 and 2. In the right column the later situation is shown, when both ribbons were linked with a new flare structure marked by arrow 3. From this moment on, both ribbons (arrow 1 and 2) became highly excited and influenced the loop footpoint area. In this area the highest RHESSI X-ray source intensity (Fig. 9) was focused. It seems that the two footpoints of the expanding loop were excited at about 9:32:21 UT.

The SDO/AIA $131 \AA$ emission intensity increased from about 9:32:21 UT, as shown by the time-slit diagram in Fig. 7. The radio source was triggered early after the start of the loop expansion, at 9:33:57 UT (horizontal solid line in Fig. 7). The earlier EUV flows are presented in panel c). This bidirectional perturbation was generated with the start time of the loop expansion (9:32:21 UT) and propagated along the slit (vertical orange solid line in the left panel of Fig. 6) with a velocity of 30 and $70 \mathrm{~km} \mathrm{~s}^{-1}$ toward the higher altitudes and the loop footpoint, respectively. Thus, the flow propagation in panel c) is significantly faster in the direction toward the footpoint.

The later EUV flows (Fig. 7, panel b) might be linked with triggering the radio source as well as with the generation of sausage magnetoacoustic waves. This bidirectional perturbation was generated just before the start time of the radio source emission (9:33:57 UT) and propagated with a velocity of 117 and $109 \mathrm{~km} \mathrm{~s}^{-1}$ toward the higher altitudes and the loop footpoint, respectively. These perturbation velocities (arrows 1 and 2) are similar to each other, and the propagation of these flows (in panel b) is significantly faster in both directions that the earlier flows (in panel c). While the earlier perturbation propagated over the entire area of the expanding loop ( $Y=280-230$ arcsec $)$, the later one was instead focused around the radio source.
The main subject of our study was the origin of the broadband (bandwidth about $1 \mathrm{GHz}$ ) sub-second pulsations (temporal period range $0.07-1.49 \mathrm{~s}$, no dominant period) that lasted $70 \mathrm{~s}$ in microwaves (about 4-7 GHz). Except for one (Fig. 4), these pulsations have no measurable frequency drift despite the high frequency range. These pulsations are not well cross-correlated at individual frequencies, and they have zero polarization. Furthermore, the equality between LCP and RCP polarization during the whole event might be caused by strong depolarization processes, for example, by a strong plasma turbulence in the radio source. This turbulent plasma scenario is supported by the facts of no radio data correlation (Fig. 2, panel a) and no characteristic dominant period in the period range $0.07-1.49 \mathrm{~s}$ (Fig. 1, bottom panel).

We tried to find the most likely possible interpretation of these pulsations in the very complex topology of the active region.

Naturally, the microwave emission observed in the solar flares is frequently linked with the gyrosynchrotron emission of mildly relativistic electrons. This incoherent radio emission is shown in panel k (Fig. 2) at frequencies $>6 \mathrm{GHz}$ (radio continuum without frequency drift). The other types of the bursts in the left panels (Fig. 2) need another explanation. We found that the radio fluxes in panels a and c (Fig. 2) correspond to the tadpole patterns shown in Figs. 3 and 5. We interpret these patterns found in the wavelet spectra of radio emission fluxes as a signatures of the fast sausage magnetoacoustic wave trains moving along a dense flare loop (waveguide) and passing through the radio source in the loop.

These magnetoacoustic wave trains are characterized by the steady periodical tail and the quasi-periodical head that can be visualized with the help of their wavelet power spectra. This characteristic wavelet tadpole pattern consists of the long-period spectral components (tadpole tail with a characteristic period $P$ ) propagating faster than the medium and short-period ones (tadpole head). Numerical simulations of these tadpole patterns can be found in Nakariakov et al. (2004) and Mészárosová et al. (2014), for instance. Observed fast sausage magnetoacoustic waves with these tadpoles were presented by Katsiyannis et al. (2003) and Mészárosová et al. (2009a,b, 2011a,b, 2013).

We detected two cases with magnetoacoustic waves (Figs. 3 and 5) and tried to use a model based on MHD oscillations (e.g., Roberts et al. 1984; Nakariakov \& Melnikov 2009). In this model the period of oscillations $\tau$ is proportional to the Alfvén transit time through the source: $\tau=2.6\left(a / v_{\mathrm{A}}\right)$, where $a$ is the small radius of the cylindrical loop. Considering the Alvén velocity $v_{\mathrm{A}}=1610 \mathrm{~km} \mathrm{~s}^{-1}$ and $\tau=0.7 \mathrm{~s}$ (Fig. 3), we obtained a very low value of $a=433.5 \mathrm{~km}$ for the radio source. It is doubtful whether this can produce our broadband pulsations. Only highly coherent plasma processes (when the brightness temperature is $T_{\mathrm{b}}=10^{14}-10^{16} \mathrm{~K}$ ) can solve this problem. Moreover, this model is commonly applied for pulsations with a period greater than $1 \mathrm{~s}$ (Nakariakov \& Melnikov 2009).

Below, we search for other arguments to explain the magnetoacoustic waves in the active region. Therefore we discuss the topology of this region in more detail.

The expanding loop (Fig. 9) consisted of some temporal fine sub-structures observed, for example, by SDO/AIA in channels $131 \AA$ channel during 9:34:33-9:35:21 UT, which are shown in Fig. 11 (processed with the NAFE method). The position of the radio source is marked by an arrow in all panels. While at 9:34:33 UT the expanding loop is very compact and simple at the site of the radio source, the later situation is different. Immediately before the radio source activity (9:34:45 UT), two 
substructures seem to be crossing in the location of the radio source. During the time of the radio source activity (9:34:57 and 9:35:09 UT), these individual sub-structures become increasingly complex and twisted (panels $b-c$ in Fig. 8 also show twisted magnetic field lines in this area). Finally, a new substructure can be seen at 9:35:21 UT (see the arrow in Fig. 11). Thus, we can postulate the hypothesis that (some of) these temporal sub-structures might be reconnected and this process could be the reason for the perturbations (bi-directional outflows) detected in the time-slit diagram (panels b-c of Fig. 7). (We note that a similar time-slit diagram, but without the separation according to individual characteristic temporal structures, was presented in Simões at al. 2015 and interpreted as the result of reconnection.) On the other hand, the actual data cadence and spatial resolution of the available observations as well as the absence of the $z$-coordinates of the event details do not allow us to make an unambiguous judgement.

The question remains why only one of the two loops was expanding. For this purpose we searched for the loop footpoint positions that might be connected with flare ribbons observed by both the SDO/AIA (panels b and d in Fig. 10) and the STEREO/SECCHI-EUVI (panels $\mathrm{e}-\mathrm{h}$ in Fig. 10) instruments. We found four such footpoints, which are marked by crosses A-D in Figs. 9 and 10. Footpoints A and B are clearly separated, both of them are connected with one of flare ribbon, and they were detected with similar radial heights (1.4 and $1.5 \mathrm{Mm})$, that is, they are located in the solar chromosphere. The situation of footpoints $\mathrm{C}$ and $\mathrm{D}$ is more complex. While footpoint $\mathrm{C}$ is connected with the most active part of the ribbon (of the highest EUV intensity), footpoint D was localized slightly aside of that ribbon part (see panel b, Fig. 10). Footpoints C and $\mathrm{D}$ were detected instead at a coronal radial hight of $3.5 \mathrm{Mm}$ and a chromospheric hight of $2.3 \mathrm{Mm}$, respectively. It seems that this very complex situation, which is connected with the ribbon marked by arrow (2) in Fig. 10 and footpoint C, might cause the loop expansion. The non-expanding loop seems to be connected instead with footpoint $\mathrm{D}$, which is localized next to the most active part of the ribbon.

The flows detected in panels $b-c$ of Fig. 7 might be linked with the magnetic reconnection outflows. We can see the flows propagating in both direction along the slit (vertical orange solid line in Fig. 6). The 131 and $94 \AA$ flows start from about the location of the radio source center at $5700 \mathrm{GHz}$ (horizontal solid line in Fig. 7 for $131 \AA$ ). Moreover, the flows in panel b) are limited to the vicinity of the radio source. (We note that the EUV time-slit diagram with reconnection inflows was studied in Yokoyama 2001). Therefore, some link between the EUV bidirectional flows and the reconnection outflows might be possible.

We suggested an explanation of the solar event observed by the different instruments at different spectral ranges. To confirm the proposed complex scenario, observational data with a higher spatial resolution are necessary.

Acknowledgements. We thank the referee for very useful comments that improved this paper. Data are courtesy of the SDO/AIA, SDO/HMI, RHESSI and STEREO/SECCHI teams. This research was supported by grants P209/12/0103 (GA CR), P209/10/1680 (GA CR), the research project RVO:67985815 of the Astronomical Institute AS, the Marie Curie PIRSES-GA-2011-295272 RadioSun project, the Slovak Research and Development Agency under the contract No. APVV-0816-11 and by the Science Grant Agency project VEGA 2/0004/16. Help of the Bilateral Mobility Project SAV-16-03 of the SAS and CAS is acknowledged. This article was created by the realization of the project ITMS No. 26220120009, based on the supporting operational Research and development program financed from the European Regional Development. I.M. acknowledges support from grants $15-02-03835$ a, 15-32$20504 \mathrm{~mol}$ a ved, and 16-32-00315 mol a. The wavelet analysis was performed with software based on tools provided by C. Torrence and G. P. Compo at http://paos. colorado. edu/research/wavelets.

\section{References}

Aschwanden, M. J. 2003, in: Proc. on Turbulence, waves and instabilities in the solar plasma, NATO advanced research workshops, NATO science series: II: mathematics, physics and chemistry, 16-20 Sept. 2002, Budapest, Hungary 124, eds. R. Erdelyi, K. Petrovay, B. Roberts, \& M. J. Awschwanden (Dordrecht: Kluwer Academic Publishers), 215

Aschwanden, M. J. 2004, Physics of Solar Corona, Chap. 15 (Berlin, Germany: Springer-Verlag)

Aschwanden, M. J., \& Benz, A. O. 1995, ApJ, 438, 997

Bogod, V. M., Alesin, A. M., \& Pervakov, A. A. 2011, Astrophys. Bull., 66, 205

Druckmüller, M. 2013, ApJS, 207, 25

Fleishman, G. D. 2004, ApJ, 601, 559

Fleishman, G. D., \& Melnikov, V. F. 1998, Sov. Phys. Uspekhi, 41, 1157

Fleishman, G. D., Stepanov, A. V., \& Yurovsky, Y. F. 1994, Sol. Phys., 153, 403 Freeland, S. L., \& Handy, B. N. 1998, Sol. Phys., 182, 497

Fu, Q. J., Gong, Y. F., Jin, S. Z., \& Zhao, R. Y. 1990, Sol. Phys., 130, 161

Grechnev, V. V., Lesovoi, S. V., Smolkov, G. Y., et al. 2003, Sol. Phys., 216, 239 Jelínek, P., \& Karlický, M. 2012, A\&A, 537, A46

Jiřička, K., Karlický, M., Mészárosová, H., \& Snížek, V. 2001, A\&A, 375, 243

Karlický, M., Zlobec, P., \& Mészárosová, H. 2010, Sol. Phys., 261, 281

Karlický, M., Jelínek, P., \& Mészárosová, H. 2011, A\&A, 529, A96

Kashapova, L. K., Tokhchukova, S. K., Rudenko, G. V., Bogod, V. M., \& Muratov, A. A. 2013a, Cent. Eur. Astrophys. Bull., 37, 573

Kashapova, L. K., Tokhchukova, S. K., Zhdanov, D. A., Bogod, V. M., \& Rudenko, G. V. 2013b, Geomagnetism and Aeronomy, 53, 1021

Katsiyannis, A. C., Williams, D. R., McAteer, R. T. J., et al. 2003, A\&A, 406, 709

Lemen, J. R., Title, A. M., Akin, D. J., et al. 2012, Sol. Phys., 275, 17 Lin, R. P., Dennis, B. R., Hurford, G. J., et al. 2002, Sol. Phys., 210, 3 Ma, Y., Xie, R., Zheng, X., \& Huang, G. 2003, Sol. Phys., 214, 353 Meshalkina, N. S., Altyntsev, A. T., Zhdanov, D. A., et al. 2012, Sol. Phys., 280, 537

Mészárosová, H., Karlický, M., Rybák, J., \& Jiřička, K. 2009a, ApJ, 697, L108 Mészárosová, H., Karlický, M., Rybák, J., \& Jiřrička, K. 2009b, A\&A, 502, L13 Mészárosová, H., Rybák, J., \& Karlický, M. 2011a, A\&A, 525, A88 Mészárosová, H., Karlický, M., \& Rybák, J. 2011b, Sol. Phys., 273, 393 Mészárosová, H., Dudík, J., Karlický, M., et al. 2013, Sol. Phys., 283, 473 Mészárosová, H., Karlický, M., Jelínek, P., \& Rybák, J. 2014, ApJ, 788, 44 Nakariakov, V. M., \& Melnikov, V. F. 2009, Space Sci. Rev., 149, 119 Nakariakov, V. M., Arber, T. D., Ault, C. E., et al. 2004, MNRAS, 349, 705 Nindos, A., Alissandrakis, C. E., Gelfreikh, G. B., et al. 1996, Sol. Phys., 165, 41

Nita, G. M., Fleishman, G. D., Kuznetsov, A. A., Kontar, E. P., \& Gary, D. E. 2015, ApJ, 799, 236

Pariiskii, I. N., Korolkov, D. V., Shivris, O. N., et al. 1976, AZh, 53, 1017 Pascoe, D. J., Nakariakov, V. M., \& Kupriyanova, E. G. 2013, A\&A, 560, A97 Roberts, B., Edwin, P. M., \& Benz, A. O. 1983, Nature, 305, 688

Roberts, B., Edwin, P. M., \& Benz, A. O. 1984, ApJ, 279, 857

Rudenko, G. V., \& Myshyakov, I. I. 2009, Sol. Phys., 257, 287

Scherrer, P. H., Schou, J., Bush, R. I., et al. 2012, Sol. Phys., 275, 207 Schou, J., Scherrer, P. H., Bush, R. I., et al. 2012, Sol. Phys., 275, 229 Simões, P. J. A., Graham, D. R., \& Fletcher, L. 2015, A\&A, 577, A68 Thompson, W. T. 2006, A\&A, 449, 791

Thompson, W. T., \& Wei, K. 2010, Sol. Phys., 261, 215

Torrence, C., \& Compo, G. P. 1998, Bull. Am. Meteorol. Soc., 79, 61 Wuelser, J.-P., Lemen, J. R., Tarbell, T. D., et al. 2004, SPIE, 5171, 111 Xie, R., Wang, M., Shi, S., et al. 2003, Chin. Astron. Astrophys., 27, 426 Yokoyama, T., Akita, K., Morimoto, T., Inoue, K., \& Newmark, J. 2001, ApJ, 546, L69

Zhdanov, D. A., \& Zandanov, V. G. 2011, Central Eur. Astrophys. Bull., 35, 223 Zhdanov, D. A., \& Zandanov, V. G. 2015, Sol. Phys., 290, 287 